\title{
Correlates of Pro-Drinking Practices in Drinking Parents of Adolescents in Hong Kong
}

\author{
Wing Man $\mathrm{Au}^{1}$, Sai Yin $\mathrm{Ho}^{1}{ }^{*}$, Man Ping Wang ${ }^{2}$, Wing Sze Lo ${ }^{1}$, Sze Pui Pamela Tin ${ }^{1}$, \\ Rong Huang ${ }^{1}$, Tai Hing Lam ${ }^{1}$
}

1 School of Public Health, The University of Hong Kong, Hong Kong, 2 School of Nursing, The University of Hong Kong, Hong Kong

* syho@ @ku.hk

\section{Abstract}

\section{Introduction and Aims}

\section{Gopenaccess}

Citation: Au WM, Ho SY, Wang MP, Lo WS, Tin SPP, Huang R, et al. (2015) Correlates of Pro-Drinking Practices in Drinking Parents of Adolescents in Hong Kong. PLOS ONE 10(3): e0119554. doi:10.1371/ journal.pone. 0119554

Academic Editor: Robert K Hills, Cardiff University, UNITED KINGDOM

Received: August 11, 2014

Accepted: January 30, 2015

Published: March 18, 2015

Copyright: $\odot 2015$ Au et al. This is an open access article distributed under the terms of the Creative Commons Attribution License, which permits unrestricted use, distribution, and reproduction in any medium, provided the original author and source are credited.

Data Availability Statement: Data cannot be made publicly available due to ethical restrictions. Data are available upon request from the Health Care and Promotion Fund by contacting syho@hku.hk.

Funding: This work was supported by the Health Care and Promotion Fund (\#25110534, to HSY, http:// www.fhb.gov.hk/en/committees/hcpf.htm). The funders took the role in approving study design, data collection and analysis but have no roles in decision to publish, or preparation of the manuscript

Competing Interests: The authors have declared that no competing interests exist.
Parental alcohol-related practices are important risk factors of adolescent drinking, but little is known about the factors associated with these parental pro-drinking practices (PPDPs). We investigated the correlates of 9 PPDPs in drinking parents of adolescents in Hong Kong.

\section{Methods}

A total of 2200 students (age 14.8 \pm 2.0 ; boys $63.2 \%$ ) participated in a school-based crosssectional survey in 2012. Analysis was restricted to 1087 (61.8\%) students with at least 1 drinking parent as PPDPs were much more common in these families. Logistic regression was used to identify correlates of each PPDP.

\section{Results}

Among 1087 students, the prevalence of PPDPs ranged from $8.2 \%$ for training drinking capacity to $65.7 \%$ for seeing parents drink. Only $14.8 \%$ of students had not experienced any of these practices. More frequent maternal drinking predicted parental training of drinking capacity. Older age predicted helping parents buy alcohol and parental encouragement of drinking. Adolescent girls were more likely to have received parental training of drinking capacity than boys. Higher perceived family affluence was associated with hearing parents saying benefits of drinking, and helping parents open bottle and pour alcohol.

\section{Conclusions}

PPDPs were associated with parental drinking frequency and various socio-demographic factors. These results have implications on alcohol control programmes involving parents to tailor messages for reducing PPDPs based on the characteristics of adolescents and parents. 


\section{Introduction}

Parental drinking is a well-known risk factor of adolescent drinking [1-4]. However, various alcohol-related parental practices may also directly or indirectly promote drinking in adolescents. For example, parental provision of alcohol and home alcohol availability were associated with adolescent drinking [5-7], and alcohol availability at home was associated with the number of alcohol-related problems experienced by adolescents [3]. A recent study also found that fetching or pouring alcohol for adults was associated with adolescent alcohol sipping [7].

We have recently identified 9 parental practices that may potentially promote alcohol drinking in Hong Kong Chinese adolescents [8]. These parental pro-drinking practices (PPDPs) include seeing parents drink and drunk; hearing parents mention the benefits of drinking and certain alcohol taste good; helping parents buy alcohol, open bottle and pour alcohol; and encouragement of drinking and training of drinking capacity (the ability to drink more without getting drunk). Exposure to these PPDPs was associated with alcohol drinking in adolescents, the results of which will be reported separately.

Studying adolescent and parental factors associated with these practices may help identify adolescents at risk of such exposures and understand reasons behind these practices. However, such studies are scarce and all based on Western populations. In an American study, mothers who drank heavily were more likely to be younger, born outside the United States, and had higher education [9]. An European study has found alcohol availability at home associated with higher family socioeconomic status [3]. However, an Australian study found no significant association between parental supply of alcohol and socio-demographic characteristics, including sex and birth order of child; and sex, household income, place of birth and religion of parents [10].

Despite alcohol use is glamorized with its purported health [11] and psychological benefits [12], the drinking prevalence in Hong Kong remains relatively low. The adolescent drinking prevalence (past 30 days) was 19\% in Hong Kong Chinese adolescents, which was much lower than that of 39\% in the United States [13, 14]. Adolescent drinking is generally regarded as inappropriate $[15,16]$ and parental influence tends to be strong in Chinese families $[17,18]$, therefore, PPDPs may have a relatively large effect on adolescent drinking in these families.

In the present study, we investigated the correlates of PPDPs in Hong Kong Chinese adolescents. We hypothesized that these practices are generally associated with parental drinking frequency and age of adolescents. Moderate alcohol drinking has been associated with higher socioeconomic status, suggesting that this group is more health conscious and may drink for the perceived benefits of alcohol [19]. Similarly, middle class parents have been reported to encourage drinking in children [20]. We, therefore, also hypothesized that positive comments about alcohol and direct encouragement of drinking are associated with higher socioeconomic status of parents.

\section{Methods}

\section{Participants and procedure}

A total of 2200 Secondary 1 (Grade 7 in the United States) to 6 students (age 14.8 \pm 2.0 , boys 63.2\%) from 4 randomly selected schools, including 2 co-education and 2 boy schools in different districts of Hong Kong were recruited for a school-based cross-sectional study in 2012. In order to recruit 4 schools, invitations to 8 schools were made with a response rate of $50 \%$. Time and administrative issues were the main reasons for school refusals. Response rate at the student level was $92 \%$ and non-participation was due to absence from school. Parents and students were informed of the survey through an invitation letter. No reply was required for participation and declining parents were to ask their children to return a blank questionnaire 
during the survey. Student participation was voluntary even with parental consent. Students completed an anonymous questionnaire independently in classrooms. Teachers were present to maintain classroom order and provide guidance based on survey instructions. Completed questionnaires were immediately collected and sealed in an opaque envelope in front of students. Ethical approval was granted by the Institutional Review Board of the University of Hong Kong/Hospital Authority Hong Kong West Cluster (UW 12-421), including consent procedure with written consent waived.

The students with missing data (mainly parental drinking status $16.8 \%$ and PPDPs $0.9 \%$ ) were excluded. To account for the differences between our sample and the underlying population in Hong Kong (Cohen effect size 0.06 for age and 0.23 for sex), the remaining 1757 students were weighted to reflect the age and sex distributions of the corresponding general population based on census data for the calculation of prevalence estimates in the present study [21]. Weighting was applied using a weighting factor determined by the sex-age combination of each subject. For example, as girls aged 16 were under-represented, these subjects were given a weighting factor of greater than 1 . As PPDPs were much more common in fami-

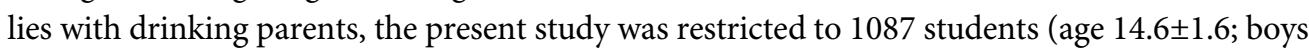
$50.2 \%)$ with at least one drinking parent.

\section{Measurements}

PPDPs. As a comprehensive list of such practices was not available in the literature, these 9 PPDPs were based on observations, discussions with adolescents and parents, and anecdotal reports. Students were asked "Have you experienced the following situations?" with response options categorized into 4 groups: 1 . Saw parents a) drink and b) being drunk; 2 . Heard parents mention a) benefits of drinking and b) alcohol tasted good; 3 . Helped parents a) buy alcohol, b) open bottle and c) pour alcohol; and 4. Parental actions a) encouraged me to drink and b) trained my drinking capacity. Students chose each option that was applicable. These pro-drinking practices in each student were analysed as individual practices and the total number of practices (0,1-2, 3-4, 5 or above).

Parental drinking. Paternal and maternal drinking were assessed by the items "How often did your father/mother drink alcohol in the past 30 days?" in two separate items each with 5 options: "never", "seldom", "sometimes", "always", "unknown".

Socio-demographic factors. Age, sex, family structure, place of birth, highest parental education and perceived family affluence were included as socio-demographic factors. Age was dichotomised as junior (11 or below to 14 ) and senior (15 or above); family structure as intact (parents together) and non-intact (divorced/live separately, mother died, father died, both died or others); and place of birth as Hong Kong and outside Hong Kong (China, Macau, Taiwan or others). Highest parental education was classified as primary or below, secondary, and tertiary. Perceived family affluence was categorized into low (relatively poor, below average), average, and high (above average, relatively rich).

\section{Statistical analysis}

Adjusted odds ratios (AORs) and 95\% confidence interval (95\% CI) were computed using STATA 10.1. Logistic regression yielded AORs and 95\% CI for each PPDP by the abovementioned socio-demographic factors adjusting for each other and school clustering. School clustering was adjusted using the command "robust clust (school variable)" in STATA 10.1. Linear regression was used to derive the regression coefficients for the number of PPDPs with similar adjustments. 


\section{Results}

Table 1 shows that after weighting, among 1087 students with one or both drinking parents, half (50.2\%) were boys, $63.2 \%$ were aged 15 or below, $80.9 \%$ were with an intact family and $74.8 \%$ were born in Hong Kong. Half the students (49.7\%) had parents with secondary education and 59.7\% reported average family affluence. Parental drinking frequency was most commonly reported to be seldom (paternal $42.8 \%$, maternal $40.1 \%$ ), followed by sometimes (paternal 27.9\%, maternal 15.3\%) and always (paternal 17.9\%, maternal 3.9\%). The prevalence of PPDPs ranged from $8.2 \%$ for training drinking capacity to $65.7 \%$ for seeing parents drink.

Only $14.8 \%$ of students had not experienced any of these practices.

Table 2 shows that seeing parents drink was negatively associated with being senior students (AOR 0.70, 95\% CI 0.52-0.94). The AOR (95\% CI) of seeing parents drink increased with paternal drinking frequency (seldom: 1.94, 1.10-3.42; sometimes: 2.10, 1.18-3.74; always: 5.06, 2.67-9.58; P for trend $<0.001$ ) but decreased with maternal drinking frequency (seldom: 1.65 , 1.21-2.25; sometimes: 1.54, 0.98-2.43; always: 0.94, 0.46-1.91; $\mathrm{P}$ for trend $<0.001$ ).

Table 3 shows that hearing the benefits of alcohol was associated with high perceived family affluence (AOR: 1.97, 95\% CI: 1.09-3.56; P for trend $=0.035$ ) and maternal drinking (seldom: $1.89,1.81-3.01$; always: 4.59, 1.91-11.00; $\mathrm{P}$ for trend $<0.001$ ). Hearing alcohol tasted good was associated with female students $(1.64,1.21-2.22)$, tertiary parental education $(1.95,1.30-2.92$; $\mathrm{P}$ for trend $=0.002$ ) and maternal drinking (seldom: 2.02, 1.46-2.78; sometimes: $2.44,1.57-$ 3.80; P for trend $<0.001$ ).

Table 4 shows that helping parents buy alcohol was associated positively with being senior students (AOR: 1.67, 95\% CI: 1.21-2.31), born outside Hong Kong $(2.18,1.57-3.02)$ and paternal drinking (sometimes: 4.01, 1.76-9.14, always: 6.55, 2.83-15.17, $\mathrm{P}$ for trend $<0.001$ ), and negatively with tertiary parental education $(0.58,0.36-0.93$; P for trend $=0.023)$. Helping parents open bottle was associated with high perceived family affluence $(1.58,1.01-2.48$; P for trend $=0.045$ ), paternal (always: 3.44, 1.81-6.53; $\mathrm{P}$ for trend $<0.001$ ) and maternal drinking (seldom: 2.11, 1.53-2.91; sometimes: 2.69, 1.72-4.19; always: 3.42, 1.70-6.89; P for trend $<0.001)$. Helping parents pour alcohol was associated with female students $(1.44,1.06-$ $1.96)$, high perceived family affluence $(1.61,1.03-2.52$; $\mathrm{P}$ for trend $=0.065)$, paternal drinking (seldom: 2.13, 1.09-4.15; sometimes: 3.29, 1.67-6.46; always: 4.63, 2.30-9.29; P for trend $<0.001$ ) and maternal drinking (seldom: 2.10, 1.54-2.89; sometimes: 2.36, 1.52-3.67; always: $3.35,1.68-6.68$; P for trend $<0.001$ ).

Table 5 shows that parental encouragement of drinking was associated positively with being senior students (AOR: 1.60, 95\% CI: 1.03-2.48), and negatively with being born outside Hong Kong $(0.59,0.36-0.98)$ and paternal drinking (seldom: 0.45, 0.21-0.95; $\mathrm{P}$ for trend $=0.208)$. Parental training of drinking capacity was associated with female students $(2.28,1.31-3.99)$ and maternal drinking (seldom: 2.18, 1.11-4.28; sometimes: 5.31, 2.52-11.19; always: 5.14, 1.8114.57; P for trend $<0.001)$.

Table 6 shows that the number of parental pro-drinking practices was associated with being female students (regression coefficient $0.26,95 \% \mathrm{CI} 0.00-0.52$ ), paternal (sometimes: 0.73 , 0.21-1.24; always: $1.46,0.92-1.99$ ) and maternal drinking (seldom: $0.75,0.50-1.02$; sometimes: $1.13,0.75-1.51$; always: $1.29,0.69-1.90)$.

\section{Discussion}

We found that PPDPs were correlated with various socio-demographic factors. Older age predicted helping parents buy alcohol and parental encouragement of drinking. It is well known that adolescent drinking increases with age [22]. Our findings also suggest a concurrent increase in parental acceptance of adolescent drinking. The law in Hong Kong does not restrict 
Table 1. Characteristics of subjects with at least one current drinking parent.

\begin{tabular}{|c|c|c|c|}
\hline Variables & Characteristics & $\mathrm{N}(\%)$ & Weighted N (\%) \\
\hline \multirow[t]{2}{*}{ Age } & Junior $(\leq 15)$ & $680(63.3)$ & 687 (63.2) \\
\hline & Senior $(\geq 16)$ & $394(36.7)$ & $400(36.8)$ \\
\hline \multirow[t]{2}{*}{ Sex } & Male & $716(66.7)$ & $546(50.2)$ \\
\hline & Female & $358(33.3)$ & $541(49.8)$ \\
\hline \multirow[t]{2}{*}{ Family structure } & Intact & $873(81.6)$ & 877 (80.9) \\
\hline & Non-intact & $197(18.4)$ & $207(19.1)$ \\
\hline \multirow[t]{2}{*}{ Place of birth } & Hong Kong & $802(75.4)$ & $805(74.8)$ \\
\hline & Others & $262(24.6)$ & $272(25.2)$ \\
\hline \multirow[t]{3}{*}{ Highest parental education } & Primary or below & $311(29.0)$ & $290(26.6)$ \\
\hline & Secondary & $539(50.2)$ & $540(49.7)$ \\
\hline & Tertiary & $224(20.9)$ & $258(23.7)$ \\
\hline \multirow[t]{3}{*}{ Perceived family affluence } & Low & $267(24.9)$ & $258(24.2)$ \\
\hline & Medium & $619(57.6)$ & $636(59.7)$ \\
\hline & High & $166(15.5)$ & $172(16.1)$ \\
\hline \multirow[t]{5}{*}{ Paternal drinking } & None & $68(6.4)$ & $85(7.9)$ \\
\hline & Seldom & $446(41.5)$ & $465(42.8)$ \\
\hline & Sometimes & $311(29.0)$ & $303(27.9)$ \\
\hline & Always & $212(19.7)$ & $194(17.9)$ \\
\hline & Unknown & $37(3.4)$ & $39(3.6)$ \\
\hline \multirow[t]{5}{*}{ Maternal drinking } & None & $439(40.9)$ & $418(38.5)$ \\
\hline & Seldom & $429(39.9)$ & $435(40.1)$ \\
\hline & Sometimes & $137(12.8)$ & $167(15.3)$ \\
\hline & Always & $44(4.1)$ & $43(3.9)$ \\
\hline & unknown & $25(2.3)$ & $24(2.2)$ \\
\hline \multirow[t]{13}{*}{ Parental pro-drinking practices } & Parents were seen & & \\
\hline & Drinking & $705(65.6)$ & $714(65.7)$ \\
\hline & Drunk & $312(29.1)$ & $317(29.1)$ \\
\hline & Parents were heard saying & & \\
\hline & Benefits of drinking & $128(11.9)$ & $138(12.7)$ \\
\hline & Alcohol tasted good & 347 (32.3) & $382(35.1)$ \\
\hline & Parents being helped & & \\
\hline & Buy alcohol & $285(26.5)$ & $287(26.4)$ \\
\hline & Open bottle & $346(32.2)$ & $331(30.4)$ \\
\hline & Pour alcohol & $356(33.1)$ & $376(34.6)$ \\
\hline & Parental actions & & \\
\hline & Encouraged drinking & $128(11.9)$ & $148(13.6)$ \\
\hline & Trained drinking capacity & $77(7.2)$ & $89(8.2)$ \\
\hline \multirow[t]{4}{*}{ Number of parental pro-drinking practices } & 0 & $172(16.0)$ & $161(14.8)$ \\
\hline & $1-2$ & $439(40.9)$ & $448(41.2)$ \\
\hline & $3-4$ & $273(25.4)$ & $273(25.2)$ \\
\hline & 5 or above & $190(17.7)$ & 204 (18.8) \\
\hline
\end{tabular}

${ }^{a}$ Weighted by age and sex of the corresponding population in Hong Kong

doi:10.1371/journal.pone.0119554.t001 
Table 2. Prevalence and adjusted odds ratios (AORs) for parents being seen drinking and drunk.

\begin{tabular}{|c|c|c|c|c|c|c|c|}
\hline \multirow[t]{2}{*}{ Characteristics } & \multirow[t]{2}{*}{ Level } & \multicolumn{3}{|c|}{ Parents were seen drinking } & \multicolumn{3}{|c|}{ Parents were seen drunk } \\
\hline & & Prevalence & AOR $^{a}$ & $95 \% \mathrm{Cl}$ & Prevalence & AOR $^{\mathrm{a}}$ & $95 \% \mathrm{Cl}$ \\
\hline \multirow[t]{2}{*}{ Age } & $\leq 15$ & 68.5 & 1 & & 28.6 & 1 & \\
\hline & $\geq 16$ & 61.0 & $0.70^{*}$ & $0.52,0.94$ & 30.0 & 1.05 & $0.77,1.44$ \\
\hline \multirow[t]{2}{*}{ Sex } & Male & 63.9 & 1 & & 30.2 & 1 & \\
\hline & Female & 67.5 & 1.21 & $0.89,1.65$ & 28.1 & 1.02 & $0.73,1.41$ \\
\hline \multirow[t]{2}{*}{ Place of birth } & Hong Kong & 64.9 & 1 & & 28.7 & 1 & \\
\hline & Others & 67.4 & 1.23 & $0.90,1.68$ & 31.4 & 1.03 & $0.74,1.43$ \\
\hline \multirow[t]{2}{*}{ Family structure } & Intact & 67.7 & 1 & & 27.7 & 1 & \\
\hline & Non-intact & 57.0 & 0.71 & $0.49,1.03$ & 35.1 & 1.25 & $0.85,1.85$ \\
\hline \multirow[t]{4}{*}{ Perceived family affluence } & Low & 56.0 & 1 & & 37.8 & 1 & \\
\hline & Medium & 72.1 & 0.94 & $0.67,1.30$ & 33.4 & 0.84 & $0.60,1.19$ \\
\hline & High & 64.9 & 0.81 & $0.52,1.26$ & 25.9 & 0.91 & $0.57,1.45$ \\
\hline & & \multicolumn{3}{|c|}{$P$ for trend $=0.225$} & \multicolumn{3}{|c|}{$P$ for trend $=0.333$} \\
\hline \multirow[t]{4}{*}{ Highest parental education } & Primary & 61.2 & 1 & & 29.3 & 1 & \\
\hline & Secondary & 68.6 & 1.30 & $0.95,1.76$ & 30.4 & 1.13 & $0.81,1.57$ \\
\hline & Tertiary & 64.7 & 1.22 & $0.82,1.80$ & 26.4 & 0.77 & $0.49,1.20$ \\
\hline & & \multicolumn{3}{|c|}{$P$ for trend $=0.117$} & \multicolumn{3}{|c|}{$P$ for trend $=0.707$} \\
\hline \multirow[t]{6}{*}{ Paternal drinking } & None & 45.5 & 1 & & 27.2 & 1 & \\
\hline & Seldom & 64.2 & $1.94 *$ & $1.10,3.42$ & 19.6 & 0.63 & $0.34,1.17$ \\
\hline & Sometimes & 65.9 & $2.10^{*}$ & $1.18,3.74$ & 29.0 & 1.11 & $0.60,2.06$ \\
\hline & Always & 79.6 & $5.06 * * *$ & $2.67,9.58$ & 52.4 & $2.87^{* *}$ & $1.52,5.41$ \\
\hline & Unknown & 58.6 & 2.08 & $0.85,5.04$ & 34.2 & 1.04 & $0.42,2.59$ \\
\hline & \multicolumn{4}{|c|}{$\mathrm{P}$ for trend (excluding unknown) $=<0.001$} & \multicolumn{3}{|c|}{$\mathrm{P}$ for trend (excluding unknown) $=<0.001$} \\
\hline \multirow[t]{6}{*}{ Maternal drinking } & None & 64.3 & 1 & & 23.0 & 1 & \\
\hline & Seldom & 67.9 & $1.65^{* *}$ & $1.21,2.25$ & 26.6 & 1.31 & $0.94,1.84$ \\
\hline & Sometimes & 67.0 & 1.54 & $0.98,2.43$ & 46.4 & $2.21^{*}$ & $1.40,3.47$ \\
\hline & Always & 64.3 & 0.94 & $0.46,1.91$ & 49.8 & $2.02 * *$ & $1.01,4.05$ \\
\hline & Unknown & 43.2 & 0.73 & $0.30,1.74$ & 25.8 & 0.94 & $0.36,2.46$ \\
\hline & \multicolumn{4}{|c|}{$P$ for trend (excluding unknown) $=0.202$} & \multicolumn{3}{|c|}{$\mathrm{P}$ for trend (excluding unknown) $=<0.001$} \\
\hline
\end{tabular}

${ }^{a}$ Adjusted odds ratios were mutually adjusted and were adjusted for school clustering effects

${ }^{*} \mathrm{p}<0.05$,

$*{ }^{*} \mathrm{p}<0.01$

$* * * p<0.001$

doi:10.1371/journal.pone.0119554.t002

children from buying alcohol in retail stores and the legal drinking age of 18 is only applicable to licensed premises such as bars and restaurants. It is unlikely that parents would encourage heavy drinking, but parents who support drinking during adolescence or early adulthood may promote drinking to prepare for their future drinking occasions [23].

Adolescent girls were more likely to have received parental training in drinking capacity. Female drunkenness is often linked to sexual harassment and unprotected sex [24, 25]. Parents might have trained drinking capacity in adolescent girls to protect them from these harms, although evidence for its effectiveness is lacking. Adolescent girls were also more likely to have heard their parents saying alcohol tasted good. Parents might have used taste to encourage girls to drink, although it is also possible that girls were more concerned about taste and hence 
Table 3. Prevalence and adjusted odds ratios (AORs) for parents being heard saying the benefits of drinking and alcohol tasted good.

\begin{tabular}{|c|c|c|c|c|c|c|c|}
\hline \multirow[t]{2}{*}{ Characteristics } & \multirow[t]{2}{*}{ Level } & \multicolumn{3}{|c|}{ Benefits of drinking } & \multicolumn{3}{|c|}{ Alcohol tasted good } \\
\hline & & Prevalence & AOR $^{a}$ & $95 \% \mathrm{Cl}$ & Prevalence & $\mathrm{AOR}^{\mathrm{a}}$ & $95 \% \mathrm{Cl}$ \\
\hline \multirow[t]{2}{*}{ Age } & $\leq 15$ & 12.8 & 1 & & 35.8 & 1 & \\
\hline & $\geq 16$ & 12.7 & 1.27 & $0.82,1.98$ & 34.0 & 1.08 & $0.80,1.47$ \\
\hline \multirow[t]{2}{*}{ Sex } & Male & 11.5 & 1 & & 28.2 & 1 & \\
\hline & Female & 14.0 & 0.96 & $0.63,1.47$ & 42.1 & $1.64 * *$ & $1.21,2.22$ \\
\hline \multirow[t]{2}{*}{ Place of birth } & Hong Kong & 13.4 & 1 & & 36.1 & 1 & \\
\hline & Others & 10.3 & 0.83 & $0.51,1.35$ & 31.4 & 0.91 & $0.65,1.25$ \\
\hline \multirow[t]{2}{*}{ Family structure } & Intact & 13.9 & 1 & & 35.5 & 1 & \\
\hline & Non-intact & 7.8 & 0.56 & $0.29,1.06$ & 33.5 & 0.86 & $0.58,1.27$ \\
\hline \multirow[t]{4}{*}{ Perceived family affluence } & Low & 10.2 & 1 & & 28.8 & 1 & \\
\hline & Medium & 11.5 & 0.93 & $0.56,1.55$ & 34.2 & 1.05 & $0.74,1.48$ \\
\hline & High & 20.6 & $1.97^{*}$ & $1.09,3.56$ & 47.3 & 1.45 & $0.93,2.27$ \\
\hline & \multicolumn{3}{|c|}{$P$ for trend $=0.035$} & \multicolumn{4}{|c|}{$P$ for trend $=0.089$} \\
\hline \multirow[t]{4}{*}{ Highest parental education } & Primary & 8.7 & 1 & & 26.7 & 1 & \\
\hline & Secondary & 10.8 & 1.38 & $0.84,2.29$ & 32.7 & 1.25 & $0.90,1.75$ \\
\hline & Tertiary & 21.3 & 1.69 & $0.95,2.98$ & 49.8 & $1.95 * *$ & $1.30,2.92$ \\
\hline & \multicolumn{3}{|c|}{$\mathrm{P}$ for trend $=0.030$} & \multicolumn{3}{|c|}{$P$ for trend $=0.002$} & \\
\hline \multirow[t]{6}{*}{ Paternal drinking } & None & 14.1 & 1 & & 52.3 & 1 & \\
\hline & Seldom & 13.1 & 0.78 & $0.37,1.68$ & 28.8 & 0.70 & $0.39,1.24$ \\
\hline & Sometimes & 13.2 & 0.98 & $0.45,2.11$ & 38.0 & 1.08 & $0.60,1.93$ \\
\hline & Always & 11.5 & 0.55 & $0.24,1.30$ & 39.9 & 1.16 & $0.63,2.13$ \\
\hline & Unknown & 5.9 & 0.47 & $0.09,2.44$ & 29.8 & 0.68 & $0.27,1.76$ \\
\hline & \multicolumn{3}{|c|}{$\mathrm{P}$ for trend (excluding unknown) $=0.337$} & \multicolumn{3}{|c|}{$P$ for trend (excluding unknown) $=0.067$} & \\
\hline \multirow[t]{6}{*}{ Maternal drinking } & None & 8.2 & 1 & & 24.6 & 1 & \\
\hline & Seldom & 14.1 & $1.89 * *$ & $1.18,3.01$ & 36.7 & $2.02 * * *$ & $1.46,2.78$ \\
\hline & Sometimes & 17.8 & 1.81 & $0.94,3.48$ & 57.2 & $2.44^{* * *}$ & $1.57,3.80$ \\
\hline & Always & 24.7 & $4.59 * *$ & $1.91,11.00$ & 45.6 & 1.85 & $0.91,3.74$ \\
\hline & Unknown & 10.0 & 1.74 & $0.37,8.10$ & 19.0 & 0.98 & $0.34,2.80$ \\
\hline & \multicolumn{3}{|c|}{$\mathrm{P}$ for trend (excluding unknown) $=<0.001$} & \multicolumn{3}{|c|}{$\mathrm{P}$ for trend (excluding unknown) $=<0.001$} & \\
\hline
\end{tabular}

${ }^{a}$ Adjusted odds ratios were mutually adjusted and were adjusted for school clustering effects

${ }^{*} \mathrm{p}<0.05$,

$*{ }^{*} \mathrm{p}<0.01$

*** $\mathrm{p}<0.001$

doi:10.1371/journal.pone.0119554.t003

could recall better than boys. Pouring alcohol for parents was also more commonly reported by girls, reflecting their greater involvement in house chores than boys [26, 27].

Supporting the hypothesis that pro-drinking practices are associated with higher socioeconomic status, higher perceived family affluence predicted hearing parents saying benefits of drinking, and helping parents open bottle and pour alcohol. Moreover, tertiary parental education also predicted parents saying alcohol tasted good. Higher socioeconomic status has been linked to health consciousness [28]. Similarly, our results suggested that parents with higher socioeconomic status tended to drink for pleasure and health, as was reported among red wine drinkers [29]. As regards family structure, students from non-intact families were less likely to report seeing parents drink, which may reflect their fewer contact hours with parents [30]. 
Table 4. Prevalence and adjusted odds ratios (AORs) for parents being helped to buy, open bottle and pour alcohol.

\begin{tabular}{|c|c|c|c|c|c|c|c|c|c|c|}
\hline \multirow[t]{2}{*}{ Characteristics } & \multirow[t]{2}{*}{ Level } & \multicolumn{3}{|c|}{ Buy alcohol } & \multicolumn{3}{|c|}{ Open bottle } & \multicolumn{3}{|c|}{ Pour alcohol } \\
\hline & & Prevalence & $A_{O R}{ }^{a}$ & $95 \% \mathrm{Cl}$ & Prevalence & $A O R^{a}$ & $95 \% \mathrm{Cl}$ & Prevalence & $A_{O R}{ }^{a}$ & $95 \% \mathrm{Cl}$ \\
\hline \multirow[t]{2}{*}{ Age } & $\leq 15$ & 22.0 & 1 & & 28.9 & 1 & & 33.0 & 1 & \\
\hline & $\geq 16$ & 34.1 & $1.67^{* *}$ & $1.21,2.31$ & 33.1 & 1.16 & $0.86,1.58$ & 37.5 & 1.22 & $0.90,1.65$ \\
\hline \multirow[t]{2}{*}{ Sex } & Male & 27.9 & 1 & & 33.3 & 1 & & 30.3 & 1 & \\
\hline & Female & 24.9 & 1.13 & $0.80,1.61$ & 27.5 & 0.86 & $0.62,1.18$ & 39.0 & $1.44^{*}$ & $1.06,1.96$ \\
\hline \multirow[t]{2}{*}{ Place of birth } & Hong Kong & 22.2 & 1 & & 30.6 & 1 & & 33.7 & 1 & \\
\hline & Others & 38.8 & $2.18^{* * *}$ & $1.57,3.02$ & 30.5 & 1.08 & $0.78,1.48$ & 36.5 & 1.14 & $0.83,1.56$ \\
\hline \multirow[t]{2}{*}{ Family structure } & Intact & 24.4 & 1 & & 30.4 & 1 & & 35.0 & 1 & \\
\hline & Non-intact & 35.4 & 1.19 & $0.79,1.77$ & 31.0 & 1.02 & $0.69,1.49$ & 33.7 & 0.86 & $0.58,1.28$ \\
\hline \multirow[t]{4}{*}{ Perceived family affluence } & Low & 32.6 & 1 & & 27.7 & 1 & & 32.9 & 1 & \\
\hline & Medium & 24.6 & 0.87 & $0.61,1.24$ & 27.2 & 1.15 & $0.82,1.63$ & 32.3 & 1.06 & $0.75,1.49$ \\
\hline & High & 23.8 & 0.84 & $0.51,1.38$ & 44.0 & $1.58^{*}$ & $1.01,2.48$ & 44.3 & $1.61^{*}$ & $1.03,2.52$ \\
\hline & \multicolumn{4}{|c|}{$P$ for trend $=0.45$} & \multicolumn{3}{|c|}{$P$ for trend $=0.045$} & \multicolumn{3}{|c|}{$P$ for trend $=0.065$} \\
\hline \multirow[t]{4}{*}{ Highest parental education } & Primary & 30.0 & 1 & & 30.0 & 1 & & 33.2 & 1 & \\
\hline & Secondary & 28.1 & 0.83 & $0.60,1.17$ & 30.6 & 1.08 & $0.78,1.49$ & 33.0 & 1.12 & $0.81,1.54$ \\
\hline & Tertiary & 18.8 & $0.58^{*}$ & $0.36,0.93$ & 30.5 & 1.14 & $0.76,1.73$ & 39.5 & 1.30 & $0.86,1.95$ \\
\hline & \multicolumn{4}{|c|}{$P$ for trend $=0.023$} & \multicolumn{3}{|c|}{$P$ for trend $=0.457$} & \multicolumn{3}{|c|}{$P$ for trend $=0.167$} \\
\hline \multirow[t]{6}{*}{ Paternal drinking } & None & 11.8 & 1 & 21.3 & 1 & & & 17.9 & 1 & \\
\hline & Seldom & 19.4 & 1.92 & $0.84,4.38$ & 22.8 & 1.21 & $0.66,2.24$ & 29.8 & $2.13^{*}$ & $1.09,4.15$ \\
\hline & Sometimes & 28.6 & $4.01 * *$ & $1.76,9.14$ & 31.8 & 1.75 & $0.94,3.24$ & 38.4 & $3.29^{* *}$ & $1.67,6.46$ \\
\hline & Always & 45.6 & $6.55^{* * *}$ & $2.83,15.17$ & 52.0 & $3.44 * * *$ & $1.81,6.53$ & 46.6 & $4.63^{* * *}$ & $2.30,9.29$ \\
\hline & Unknown & 27.5 & 2.37 & $0.79,7.10$ & 21.3 & 0.99 & $0.38,2.58$ & 35.4 & 2.54 & $0.96,6.69$ \\
\hline & \multicolumn{4}{|c|}{$\mathrm{P}$ for trend (excluding unknown) $=<0.001$} & \multicolumn{3}{|c|}{$\begin{array}{l}\mathrm{P} \text { for trend (excluding unknown) }= \\
<0.001\end{array}$} & \multicolumn{3}{|c|}{$\begin{array}{l}\text { P for trend (excluding unknown) }= \\
<0.001\end{array}$} \\
\hline \multirow[t]{6}{*}{ Maternal drinking } & None & 22.7 & 1 & & 21.8 & 1 & & 24.5 & 1 & \\
\hline & Seldom & 24.3 & 1.41 & $1.00,2.00$ & 34.1 & $2.11 * * *$ & $1.53,2.91$ & 38.9 & $2.10^{* * *}$ & $1.54,2.89$ \\
\hline & Sometimes & 35.2 & 1.47 & $0.91,2.40$ & 37.9 & $2.69^{* * *}$ & $1.72,4.19$ & 45.3 & $2.36^{* * *}$ & $1.52,3.67$ \\
\hline & Always & 39.1 & 1.88 & $0.91,3.89$ & 55.6 & $3.42^{* *}$ & $1.70,6.89$ & 59.1 & $3.35^{* *}$ & $1.68,6.68$ \\
\hline & Unknown & 45.3 & 1.49 & $0.60,3.71$ & 15.6 & 0.55 & $0.18,1.71$ & 15.4 & 0.60 & $0.19,1.85$ \\
\hline & \multicolumn{4}{|c|}{$\mathrm{P}$ for trend (excluding unknown) $=0.045$} & \multicolumn{3}{|c|}{$\begin{array}{l}\mathrm{P} \text { for trend (excluding unknown) }= \\
\qquad<0.001\end{array}$} & \multicolumn{3}{|c|}{$\begin{array}{l}\mathrm{P} \text { for trend (excluding unknown) }= \\
<0.001\end{array}$} \\
\hline
\end{tabular}

${ }^{a}$ Adjusted odds ratios were mutually adjusted and were adjusted for school clustering effects

${ }^{*} \mathrm{p}<0.05$,

${ }^{* *} \mathrm{p}<0.01$,

$* * * \mathrm{p}<0.001$

doi:10.1371/journal.pone.0119554.t004

Students born outside Hong Kong, mainly from Mainland China, were more likely to report helping parents buy alcohol. The type of alcohol bought was not recorded but beer or Chinese wines seemed more likely as they would be easier for adolescents to identify and buy than the vast variety of Western wines and spirits. This speculation is consistent with the beer drinking preference of people with lower socioeconomic status that is more common among new immigrants from the Mainland [31]. These parents were less likely to encourage adolescent drinking, suggesting that health and social status were not their main reasons for drinking.

The results of the present study would be useful for family-based and school-based alcohol control programmes involving parents to tailor contents targeting various PPDPs according to the socio-demographic characteristics of students (age, sex, place of birth) and parents (education level, socioeconomic status). Our results also provide support for persuading parents to drink less, not to drink in front of children and not to involve them in buying alcohol, opening 
Table 5. Prevalence and adjusted odds ratios (AORs) for parental encouragement of drinking and training of drinking capacity.

\begin{tabular}{|c|c|c|c|c|c|c|c|}
\hline \multirow[t]{2}{*}{ Characteristics } & \multirow[t]{2}{*}{ Level } & \multicolumn{3}{|c|}{ Parental encouragement of drinking } & \multicolumn{3}{|c|}{ Parental training of drinking capacity } \\
\hline & & Prevalence & AOR $^{a}$ & $95 \% \mathrm{Cl}$ & Prevalence & AOR $^{a}$ & $95 \% \mathrm{Cl}$ \\
\hline \multirow[t]{2}{*}{ Age } & $\leq 15$ & 12.7 & 1 & & 8.7 & 1 & \\
\hline & $\geq 16$ & 15.0 & $1.60^{*}$ & $1.03,2.48$ & 7.3 & 0.77 & $0.43,1.36$ \\
\hline \multirow[t]{2}{*}{ Sex } & Male & 12.4 & 1 & & 5.9 & 1 & \\
\hline & Female & 14.8 & 1.03 & $0.66,1.61$ & 10.5 & $2.28^{* *}$ & $1.31,3.99$ \\
\hline \multirow[t]{2}{*}{ Place of birth } & Hong Kong & 14.7 & 1 & & 8.8 & 1 & \\
\hline & Others & 9.9 & $0.59 *$ & $0.36,0.98$ & 5.9 & 0.58 & $0.30,1.11$ \\
\hline \multirow[t]{2}{*}{ Family structure } & Intact & 12.9 & 1 & & 7.3 & 1 & \\
\hline & Non-intact & 16.4 & 1.33 & $0.78,2.24$ & 11.9 & 1.79 & $0.97,3.32$ \\
\hline \multirow[t]{4}{*}{ Perceived family affluence } & Low & 13.7 & 1 & & 7.3 & 1 & \\
\hline & Medium & 12.1 & 0.70 & $0.44,1.12$ & 6.3 & 0.95 & $0.50,1.82$ \\
\hline & High & 17.5 & 1.09 & $0.60,1.97$ & 15.8 & 1.62 & $0.77,3.42$ \\
\hline & \multicolumn{2}{|c|}{$\mathrm{P}$ for trend $=0.840$} & & & \multicolumn{2}{|c|}{$P$ for trend $=0.172$} & \\
\hline \multirow[t]{4}{*}{ Highest parental education } & Primary & 10.2 & 1 & & 7.2 & 1 & \\
\hline & Secondary & 13.4 & 1.30 & $0.80,2.11$ & 7.1 & 0.89 & $0.46,1.59$ \\
\hline & Tertiary & 17.7 & 1.25 & $0.69,2.26$ & 11.6 & 1.28 & $0.64,2.54$ \\
\hline & \multicolumn{2}{|c|}{$P$ for trend $=0.392$} & & & \multicolumn{2}{|c|}{$\mathrm{P}$ for trend $=0.358$} & \\
\hline \multirow[t]{6}{*}{ Paternal drinking } & None & 35.7 & 1 & & 10.5 & 1 & \\
\hline & Seldom & 8.3 & $0.45^{*}$ & $0.21,0.95$ & 7.7 & 1.14 & $0.43,3.04$ \\
\hline & Sometimes & 13.0 & 0.72 & $0.34,1.52$ & 6.7 & 1.01 & $0.37,2.73$ \\
\hline & Always & 17.6 & 0.75 & $0.34,1.65$ & 11.4 & 1.53 & $0.55,4.26$ \\
\hline & Unknown & 12.7 & 0.28 & $0.07,1.17$ & 5.4 & 0.37 & $0.06,2.13$ \\
\hline & \multicolumn{3}{|c|}{$P$ for trend (excluding unknown) $=0.208$} & & \multicolumn{3}{|c|}{$P$ for trend (excluding unknown) $=0.493$} \\
\hline \multirow[t]{6}{*}{ Maternal drinking } & None & 8.8 & 1 & & 3.9 & 1 & \\
\hline & Seldom & 12.6 & 1.16 & $0.72,1.86$ & 8.6 & $2.18^{*}$ & $1.11,4.28$ \\
\hline & Sometimes & 24.7 & 1.62 & $0.89,2.96$ & 16.1 & $5.31 * * *$ & $2.52,11.19$ \\
\hline & Always & 30.5 & 2.06 & $0.87,4.89$ & 19.5 & $5.14 * *$ & $1.81,14.57$ \\
\hline & Unknown & 7.2 & 0.37 & $0.05,2.89$ & 0 & - & - \\
\hline & \multicolumn{3}{|c|}{$P$ for trend (excluding unknown) $=0.010$} & & \multicolumn{3}{|c|}{$\mathrm{P}$ for trend $=<0.001$} \\
\hline
\end{tabular}

${ }^{a}$ Adjusted odds ratios were mutually adjusted and were adjusted for school clustering effects

${ }^{*} \mathrm{p}<0.05$,

${ }^{* *} \mathrm{p}<0.01$

*** $\mathrm{p}<0.001$

doi:10.1371/journal.pone.0119554.t005

bottles and pouring alcohol. The present study would be particularly relevant also to Mainland Chinese parents who share similar cultural backgrounds.

Our study has several limitations. All data were reported by students including PPDPs and parental drinking, although adolescent reports were found to correlate with parental drinking practices [32]. Moreover, for social desirability, parents may tend to under-report their drinking-related practices, but adolescents would have little motivation in doing so. Having information on alcohol types would have enhanced the interpretation of results although it would not alter the conclusions of the present study. Finally, the cross-sectional correlations identified do not imply causal effects. 
Table 6. Mean (SD) and regression coefficient on the number of parental pro-drinking practices.

\begin{tabular}{|c|c|c|c|c|}
\hline \multirow[t]{2}{*}{ Characteristics } & \multirow[t]{2}{*}{ Level } & \multicolumn{3}{|c|}{ Number of parental pro-drinking practices } \\
\hline & & Mean (SD) & Regression coefficient $^{a}$ & $95 \% \mathrm{Cl}$ \\
\hline \multirow[t]{2}{*}{ Age } & $\leq 15$ & $2.51(1.98)$ & Reference & \\
\hline & $\geq 16$ & $2.65(2.03)$ & 0.16 & $-0.09,0.42$ \\
\hline \multirow[t]{2}{*}{ Sex } & Male & $2.44(2.07)$ & Reference & \\
\hline & Female & $2.68(1.91)$ & $0.26^{*}$ & $0.00,0.52$ \\
\hline \multirow[t]{2}{*}{ Place of birth } & Hong Kong & $2.53(2.03)$ & Reference & \\
\hline & Others & $2.62(1.89)$ & 0.13 & $-0.13,0.39$ \\
\hline \multirow[t]{2}{*}{ Family structure } & Intact & $2.55(1.98)$ & Reference & \\
\hline & Non-intact & $2.62(2.09)$ & -0.04 & $-0.36,0.28$ \\
\hline \multirow[t]{4}{*}{ Perceived family affluence } & Low & $2.56(1.84)$ & Reference & \\
\hline & Medium & $2.39(1.92)$ & -0.07 & $-0.35,0.21$ \\
\hline & High & $3.11(2.21)$ & 0.32 & $-0.05,0.70$ \\
\hline & & & $P$ for trend $=0.074$ & \\
\hline \multirow[t]{4}{*}{ Highest parental education } & Primary & $2.36(2.06)$ & Reference & \\
\hline & Secondary & $2.55(1.90)$ & 0.17 & $-0.10,0.43$ \\
\hline & Tertiary & $2.80(2.09)$ & 0.22 & $-0.11,56$ \\
\hline & & & $P$ for trend $=0.123$ & \\
\hline \multirow[t]{6}{*}{ Paternal drinking } & None & $2.36(1.68)$ & Reference & \\
\hline & Seldom & $2.14(1.87)$ & 0.18 & $-0.32,0.69$ \\
\hline & Sometimes & $2.65(2.03)$ & $0.73^{* *}$ & $0.21,1.24$ \\
\hline & Always & $3.57(2.06)$ & $1.46 * * *$ & $0.92,1.99$ \\
\hline & Unknown & $2.31(1.57)$ & 0.12 & $-0.67,0.90$ \\
\hline & & & $\mathrm{P}$ for trend $<0.001$ (excluding unknown) & \\
\hline \multirow[t]{6}{*}{ Maternal drinking } & None & $2.02(1.77)$ & Reference & \\
\hline & Seldom & $2.64(1.93)$ & $0.76 * * *$ & $0.50,1.02$ \\
\hline & Sometimes & $3.48(2.05)$ & $1.13^{* * *}$ & $0.75,1.51$ \\
\hline & Always & $3.88(2.52)$ & $1.29 * * *$ & $0.69,1.90$ \\
\hline & Unknown & $1.81(1.84)$ & -0.26 & $-1.03,0.52$ \\
\hline & & & $P$ for trend $<0.001$ (excluding unknown) & \\
\hline
\end{tabular}

${ }^{a}$ Regression coefficients were adjusted for other factors shown in table and school clustering effects

doi:10.1371/journal.pone.0119554.t006

\section{Conclusions}

PPDPs were associated with parental drinking frequency and various socio-demographic factors. These results have implications on alcohol control programmes involving parents to tailor messages for reducing PPDPs based on the characteristics of adolescents and parents.

\section{Acknowledgments}

We would like to thank the subjects who participated in the surveys.

\section{Author Contributions}

Conceived and designed the experiments: WMA SYH MPW WSL SPPT RH THL. Performed the experiments: WMA. Analyzed the data: WMA SYH. Contributed reagents/materials/analysis tools: SYH THL. Wrote the paper: WMA SYH MPW WSL SPPT RH THL. 


\section{References}

1. Van Der Vorst H, Engels RCME, Meeus W, Deković M, Van Leeuwe J. The role of alcohol-specific socialization in adolescents' drinking behaviour. Addiction. 2005; 100(10): 1464-1476. PMID: 16185208

2. Ennett ST, Bauman KE, Foshee VA, Pemberton M, Hicks KA. Parent-child communication about adolescent tobacco and alcohol use: what do parents say and does it affect youth behavior? J Marriage Fam. 2001; 63(1): 48-62.

3. Spijkerman R, Van Den Eijnden RJJM, Huiberts A. Socioeconomic differences in alcohol-specific parenting practices and adolescents' drinking patterns. Eur Addict Res. 2008; 14(1): 26-37. doi: 10.1159/ 000110408 PMID: 18182770

4. Mares SH, Van Der Vorst H, Engels RCME, Lichtwarck-Aschoff A. Parental alcohol use, alcohol-related problems, and alcohol-specific attitudes, alcohol-specific communication, and adolescent excessive alcohol use and alcohol-related problems: An indirect path model. Addict Behav. 2011; 36(3): 209-216. doi: 10.1016/j.addbeh.2010.10.013 PMID: 21084165

5. Altman D, Wolfson M. Adults' approval and adolescents' alcohol use. J Adolesc Health. 2004; 35(4): 345-354. PMID: 15830447

6. Komro KA, Maldonado-Molina MM, Tobler AL, Bonds JR, Muller KE. Effects of home access and availability of alcohol on young adolescents' alcohol use. Addiction. 2007; 102(10): 1597-1608. PMID: 17854336

7. Jackson C, Ennett ST, Dickinson DM, Bowling JM. Attributes that differentiate children who sip alcohol from abstinent peers. J Youth Adolesc. 2013; 42(11): 1687-1695. doi: 10.1007/s10964-012-9870-8 PMID: 23224982

8. Au WM, Ho SY, Wang MP, Lo WS, Tin SPP, et al. Alcohol drinking and pro-drinking practices in parents of Hong Kong adolescents. Alcohol Alcohol. 2014; 49(6): 668-674. doi: 10.1093/alcalc/agu063 PMID: 25288615

9. Niyonsenga T, Rojas P, Dillon F, Kim S, Ganapati EN, et al. Correlates of heavy drinking behaviors of Latino mothers and their adult daughters. J Psychoactive Drugs. 2010; 42(4): 457-466. PMID: 21305910

10. Ward BM, Snow PC. Factors affecting parental supply of alcohol to underage adolescents. Drug Alcohol Rev. 2011; 30(4): 338-343. doi: 10.1111/j.1465-3362.2010.00228.x PMID: 21355899

11. Stampfer MJ, Colditz GA, Willett WC, Speizer FE, Hennekens $\mathrm{CH}$. A prospective study of moderate alcohol consumption and the risk of coronary disease and stroke in women. N Engl J Med. 1988; 319(5): 267-273. PMID: 3393181

12. Baum-Baicker $C$. The psychological benefits of moderate alcohol consumption: a review of the literature. Drug Alcohol Depend. 1985; 15(4): 305-322. PMID: 4053968

13. Eaton DK, Kann L, Kinchen S, Shanklin S, Flint KH, et al. Youth risk behavior surveillance-United States, 2011. MMWR Surveill Summ. 2012; 61(4): 1-162. PMID: 22673000

14. Li R, Wong $P$, editors. The $2011 / 12$ survey of drug use among students [Internet]. Hong Kong; 2013 [cited 2014 June 28]. Available from: http://www.nd.gov.hk/en/survey_of_drug_use_11-12.htm

15. Gruber E, Diclemente RJ, Anderson MM, Lodico M. Early drinking onset and its association with alcohol use and problem behavior in late adolescence. Prev Med. 1996; 25(3): 293-300. PMID: 8781007

16. Swahn MH, Simon TR, Hammig BJ, Guerrero JL. Alcohol-consumption behaviors and risk for physical fighting and injuries among adolescent drinkers. Addict Behav. 2004; 29(5): 959-963. PMID: 15219342

17. Yau OH. Chinese cultural values: their dimensions and marketing implications. Eur J Mark. 1988; 22(5): 44-57.

18. Lee E, Mock MR. Chinese families: Ethnicity and family therapy. New York: Guilford Press; 1996. 302-318 p.

19. Huckle T, You RQ, Casswell S. Socio-economic status predicts drinking patterns but not alcohol-related consequences independently. Addiction. 2010; 105(7): 1192-1202. doi: 10.1111/j.1360-0443. 2010.02931.x PMID: 20456295

20. Clark L, editor. Middle-class parents blamed for rise in binge-drinking by encouraging children to drink alcohol at home [Internet]; 2008 [cited 2014 November 15]. Available from http://www. dailymail.co.uk/ news/article-1081851/Middle-class-parents-blamed-rise-binge-drinking-encouraging-children-drinkalcohol-home.html.

21. Census and Statistics Department. Hong Kong annual digest of Statistics [Internet]. Hong Kong; 2012 [cited 2014 June 28]. Available from https://www.censtatd.gov.hk/hkstat/sub/sp140.jsp? productCode= B1010003.accessed. 
22. Wang MP, Ho SY, Lam TH. Underage alcohol drinking and medical services use in Hong Kong: a cross-sectional study. BMJ Open. 2013; 3(5): e002740. doi: 10.1136/bmjopen-2013-002740 PMID 23793697

23. Boyle JR, Boekeloo BO. Perceived parental approval of drinking and its impact on problem drinking behaviors among first-year college students. J Am Coll Health. 2006; 54(4): 238-244. PMID: 16450849

24. Strunin L, Hingson R. Alcohol, drugs, and adolescent sexual behavior. Subst Use Misuse. 1992; 27(2): 129-146.

25. Bonomo $\mathrm{Y}$, Coffey $\mathrm{C}$, Wolfe R, Lynskey M, Bowes G, et al. Adverse outcomes of alcohol use in adolescents. Addiction. 2001; 96(10): 1485-1496. PMID: 11571067

26. Bond MHE. The handbook of Chinese psychology: Oxford University Press; 1996.

27. Hoschild A, Machung A. The second shift: Working parents and the revolution at home. New York, USA: Viking; 1989.

28. Wardle J, Steptoe A. Socioeconomic differences in attitudes and beliefs about healthy lifestyles. J Epidemiol Community Health. 2003; 57(6): 440-443. PMID: 12775791

29. Cox D. Predicting consumption, wine involvement and perceived quality of Australian red wine. J Wine Res. 2009; 20(3): 209-229.

30. Sandberg JF, Hofferth SL. Changes in children's time with parents: United States, 1981-1997. Demography. 2001; 38(3): 423-436. PMID: 11523269

31. Janghorbani M, Ho SY, Lam TH, Janus ED. Prevalence and correlates of alcohol use: a populationbased study in Hong Kong. Addiction. 2003; 98(2): 215-224. PMID: 12534427

32. O'malley SS, Carey KB, Maisto SA. Validity of young adults' reports of parental drinking practices. J Stud Alcohol Drugs. 1986; 47(5): 433. PMID: 3762165 